\title{
What Do Educators Need To Know To Promote Student Performance On Dual Tasks?
}

\author{
Genevieve Pinto Zipp, Seton Hall University, USA
}

\begin{abstract}
In today's society, doing two things at once is the norm. As educators, especially in the area of physical education and special education, we assist students in performing multiple motor tasks successfully at one time. Understanding how the performance of multiple tasks affects the success of the overall performance may provide educators with knowledge regarding the most effective and efficient learning strategies to be used when teaching dual task performance. Therefore, this study was designed to provide insight by comparing the effects of performing dual tasks requiring different attentional demands on walking parameters in 6- and 7-year old children. It was hypothesized that a task, which requires a higher degree of attentional demands, would cause a disturbance of the children's gait, with greater changes noted in the younger children.
\end{abstract}

The subject population comprised of 13 boys, six 7-year olds (mean age $=7$ yrs, 4 mos) and seven 6-year olds (mean age $=6$ yrs, 6 mos) with no known medical problems.

The subjects in both age groups walked at self-selected velocities over the Gait Rite Gold mat which is a 4 meter by 0.5 meter computerized walkway designed to capture spatial and temporal parameters of gait. Each subject walked under four conditions, with four trials per condition. The conditions were: 1) self-paced walking, 2) self-paced walking holding a 3/4 full, open plastic pitcher of water, 3) self-paced walking carrying a knapsack containing weights totaling 15 lbs., and 4) self-paced walking while performing a cognitive task requiring a color/action association verbal response. Conditions 2 and 4 were considered the high attentional demand tasks. The order of the conditions were randomized and counterbalanced across the subjects. Data collection started as the subject stepped onto the mat and ended when the subject stepped off the mat.

Multi-factorial ANOVAs on the dependent variables of velocity and cadence, with leg length as the covariant were performed ( $p \leq .05)$. Scheffe post hoc analyses were used to compare individual means. The SPSS program - 11.5 for Windows - was used to perform all the calculations.

When looking at conditions, a main effect was noted for velocity $p \leq .001$ and cadence $p \leq .049$. However, there was no main effect observed for age or age-by-condition interaction. Scheffe post hoc analysis revealed a significant difference in cadence when walking with the water versus selfpaced walking only $p \leq .001$; for velocity when walking with water versus walking with the knapsack, a trend was noted, $p \leq .07$.

The findings of this study support the hypothesis that performing a secondary dual task that requires a high degree of attention (walking with water) will have a greater effect on the primary task of gait as noted by gait parameter changes. Interestingly, age did not significantly influence gait changes in these healthy young children.

In today's fast-paced world, doing several things at once is common. For example, walking while talking on a cell phone and crossing a busy street is a common occurrence, even for young children. The issue of how safe is one when doing multiple things at once frequently emerges in the news. Educators must begin to examine the effects of dual task performance on the safety and 
quality of the performance so that they can begin to help students focus their attention on the relevant features in the environment, which must be monitored. The findings of this study, as well as others, suggest that incorporating a dual task requirement during walking may provide an individual the opportunity to develop and practice movement strategies required in performing everyday activities regardless of age, and it affords insight into learning strategies that educators may use.

Keywords: Performing Dual Tasks; Dual Task Strategies; Promoting Learning

\section{INTRODUCTION}

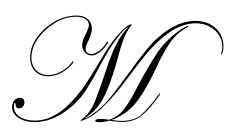

any common activities require simultaneous performance of more than one task. Walking is a good example; many times walking is paired with a wide range of activities or tasks that require varying levels of attention, such as carrying on a conversation while walking or carrying an object. The concurrent performance of these activities is subject to the attentional demands required by each of the tasks. It is possible that interference between the tasks can occur, especially if they are using the same processing resources Magill ${ }^{1}$. Although, under normal conditions, walking is automatic it is still subject to environmental demands. Shumway-Cook and Woollacott ${ }^{2}$ found that healthy older adults had difficulty maintaining postural control when faced with attentionally demanding situations. O'Shea et al. ${ }^{3}$, Morris etal. ${ }^{4}$, and Bond and Morris ${ }^{5}$ demonstrated diminished gait performance in both Parkinson patients and the healthy elderly population when engaged in performing dual tasks, regardless of the attentional demands required. Camicioli et. al. ${ }^{6}$ further demonstrated the effects of dual task performance on gait parameters in Alzheimer patients. Interestingly, the effects of dual task performance has not been well documented in children, thus we cannot assume that the affects noted in adults can be generalized to children.

Magill ${ }^{1}$ states that attentional focus incorporates both width and direction - width, meaning a broad or narrow focus on the environmental constraints, and direction referring to whether the attention is focused on external or internal cues in the environment. For example, a situation could require a broad/external focus or a narrow/internal focus. A broad external focus would be used in navigating a crowded school corridor, while cognitive tasks (problem-solving tasks, such as math computations) require an internal focus. Magill ${ }^{1}$ suggests that people are capable of switching focus depending on the demands of the environment. Yet, this switching can have a detrimental effect on the performance of the activity by effecting timing or coordination. Wickens ${ }^{7}$ suggests that when performing two tasks that share a common resource, the tasks will be performed less well than if the two tasks were competing for different resources. In an activity, such as walking while carrying a container of water, the increased attentional demands associated with the water may shift the available cognitive resources. Thus, the shift may affect the performance of the secondary task. This can result in a disruption of an "automatic" action or the use of a less efficient strategy ${ }^{8}$. In a study involving acquired brain injured subjects and age-matched normal's, the effect of performing a cognitive secondary task on a primary motor task were assessed (Haggard et al, 2000) ${ }^{9}$. The study demonstrated that the increased interference caused by the high cognitive demanding task resulted in a decrease in the automatic performance of the motor task for the acquired brain injured subjects. Haggard et al $(2000)^{9}$ referred to this deterioration as the "dual task decrement". Interestingly, Haggard et al (2000) also noted for several of their age-matched normal's that the cognitive element of the task was prioritized at the expense of the motor task (walking pattern).

While numerous investigation have been undertaken to further access the effects of dual tasks on motor performance, limited work has been conducted on multifaceted tasks that are more functional in nature and may require a higher degree of attentional demands (Bond and Morris, 2000) ${ }^{5}$. Furthermore, current investigations have only utilized healthy and neurologically impaired adults, thereby limiting generalizability of the findings to children at this time. Therefore, the purpose of this study was to determine the effects of performing multifaceted dual tasks on gait parameters in children. The hypothesis was that a secondary task requiring a high degree of attention would influence gait performance to a greater degree than a less demanding task. A second question addressed whether a difference in performance was related to age. 


\section{METHODS}

\section{Subjects}

The subjects were 13 boys - six 7-year olds (mean age 7 yrs., 4 mos.) and seven 6-year olds (mean age 6 yrs., 6 mos.). All subjects were in good health with no known motor impairments or medical problems. This study was approved by the Seton Hall University IRB and written parental consent for minor participation was obtained.

\section{Apparatus}

Data were collected using the GAIT RITE GOLD tm system; the gait rite mat is $4 \mathrm{~m}$ long and $.5 \mathrm{~m}$ wide. Data are collected by sensors in the mat and recorded in the unit's software program. Collection of the data began as the subject stepped onto the mat and ended when the subject stepped off the mat. The starting line for the initiation of walking was $10 \mathrm{ft}$. prior to the mat to ensure that the subject's initial steps on the mat were consistent, and the finish line, which signified the end of the subjects' walking trial, was $10 \mathrm{ft}$. beyond the end of the mat to encourage the subjects to maintain their gait pattern past the end of the mat.

Three conditions requiring differing amounts of attention were utilized in the study. These conditions included walking while carrying a container of water (WW - high attentional demands), walking while carrying a knapsack (WNAP - low attentional demands), and walking while performing a cognitive task (WCOG - high attentional demands). The knapsack was canvas and weighed 15 pounds. The container for the water was a 4-cup plastic measuring cup, which was filled 3/4 full. The cognitive task used three pieces of construction paper differing in color - red, yellow and green.

\section{Procedure}

The subjects performed each of the four conditions four times in a random and counterbalanced fashion, resulting in 16 trials total, thus addressing any potential ordering effects due to condition presentation. Prior to starting the series of trials, each subject's height and lower leg length were measured using a standard tape measure. The subjects' body weights were obtained from their legal guardians. General instructions were given before each trial, which were: 1) When walking, do not step on the data cases along the edge of the mat, 2) begin walking at the starting line, and 3) continue walking until you reach the finish line. The subjects were also given instructions that pertained to each condition. For all conditions, the subjects were asked to walk at their normal pace (i.e., as if they were walking down the hall in school). While walking and carrying the container of water, the subjects were instructed that the goal was to walk at their normal pace while not spilling the water. During the knapsack condition, the subjects were instructed to again walk at their preferred pace while carrying the knapsack on their right shoulder only, using any holding pattern. For the cognitive condition, the subjects were instructed to verbally respond with the name of the action associated with the color of the paper being held up by the examiner (red $=$ stop, yellow $=$ slow, and green $=$ go). The papers were hidden from view and one paper was presented in a random fashion during each of the trials. When the paper was presented, the subject was asked to verbally respond while maintaining his walking pace with no regard to the verbal response that they made. In order to determine the amount of interference caused by the secondary task, two gait parameters were measured - velocity and cadence.

\section{RESULTS}

Multi-factorial repeated measures ANOVAs (2 groups $x 4$ conditions), on the dependent variables of velocity and cadence, were carried out with the participant's leg length as the covariant $(\mathrm{p} \leq .05)$. Scheffe post hoc analyses were used to compare individual means. Levene's tests for homogeneity of variance showed that the differences in variance were not statistically significant. The SPSS 11.0 for windows program was used to perform all the calculations.

When looking at conditions, main effects were noted for cadence $\mathrm{p} \leq .049$ and velocity $\mathrm{p} \leq .001$. However, there was no main effect observed for age or an age-by-condition interaction. Scheffe post hoc analysis revealed a significant difference in cadence when walking with the water versus normal walking $(\mathrm{p} \leq .001)$ and for velocity when walking with water versus walking with the knapsack, a trend was noted $\mathrm{p} \leq .07$. 


\section{DISCUSSION/CONCLUSION}

Although it was expected that age would impact performance, no difference was found between the 6- and 7-year olds. This may be related to the small sample size and the limited age range chosen. Differences in motor performance may be observed when comparing 7-year olds to either younger children (5 years of age or less) or older children ( 9 years or older) as a result of more apparent differences in cognitive capabilities and motor abilities across these age ranges. However, the results of this study do lend support to the assumption that tasks requiring high attentional demands affect gait parameters to a greater degree when compared to tasks requiring less attention. The high attention demanding task of walking with the water required the subjects to decrease their velocity and cadence in an attempt to successfully meet the task goal, thereby demonstrating the dual task decrement suggested by Haggard et al (2000) ${ }^{9}$. To the surprise of the author, the cognitive condition task did not significantly impact gait parameters. One explanation may be that this specific task reflects a well learned response, which is an intrinsic component of our daily life, even for young children who apparently have transitioned from the early stage of learning this task to the later stage of learning. If the task was substituted with a less well learned cognitive task, the question remains as to whether a difference in performance would have emerged.

The knowledge obtained from this study supports the existence of the dual task decrement and suggests the importance of identifying the potential interference that may occur as a result of performing dual tasks. Understanding these effects may assist in structuring more effective and efficient learning sessions, especially in the area of physical education and special education where the performance of multiple motor tasks is fundamental in the environment. Learning sessions that solely focus on single task performance may not transfer to dual task situations effectively. Furthermore, training sessions utilizing dual tasks that promote minimal interference may affect motor performance to a lesser degree than those that promote a higher degree of interference.

It is important that educators teaching motor skill acquisition incorporate the findings from dual task investigations when teaching students dual tasks. Utilizing the full body of knowledge, which incorporates environmental factors, task characteristics and learning capabilities, will aide educators of motor skills in identifying effective strategies and eliminating learning strategies that are not helping students complete dual tasks safely and efficiently.

\section{AUTHOR INFORMATION}

Dr. Pinto Zipp is an Associate Professor, Department of Graduate Programs in Health Sciences at Seton Hall University. She received her Ed. D. from Teachers College Columbia University in 1996. She currently teaches management of neuromuscular problems in the Doctor of Physical Therapy program. Her research interests focus on, a) effects of performing dual tasks on walking performance and postural sway in children and adults, and b) curriculum design issues including the use of mind mapping and video based cases in professional education for the promotion of clinical decision making skills. E-mail: genevieve.zipp@shu.edu

\section{REFERENCES}

1. Magill, R. A. Motor Learning Concepts and Applications. Boston: WCB Mcgraw-Hill.1998:101-113.

2. Shumway-Cook, A. Woollacott, M. Attentional demands and postural control: the effect of sensory context. Journal of gerontology: Medical Sciences. 2000: 55A (1): M10-M16.

3. O'Shea, S., Morris, M. E., Iansek, R. Dual task interference during gait in people with Parkinson disease: effects of motor versus cognitive secondary tasks. Physical Therapy. 2002; 82 (9):888-897.

4. Morris, M. E., et al. Stride length regulation in Parkinson's disease normalization strategies and underlying mechanisms. Brain.1996; 119: 551-568.

5. Bond, J. M., Morris, M. Goal directed secondary motor tasks: their effects on gait in subjects with Parkinson disease. Archives of Physical Medicine and Rehabilitation. 2000; 81: 110-116.

6. Camicioli, R., et al. Talking while walking: the effect of a dual task in aging and Alzheimer's disease. Neurology. 1997; (April) 48: 955-958.

7. Wickens, C. D. Engineering Psychology and Human Performance. $\left(2^{\text {nd }}\right.$ ed.) New York: Harper Collins, 1992. 
8. Hockey, G. R. J. Compensatory control in the regulation of human performance under stress and high workload: a cognitive-energetical framework. Biological Psychology.1997; 45: 73-93.

9. Haggard, $\mathrm{P}$, et al. Interference between gait and cognitive tasks in a rehabilitating neurological population. Neurol Neurosurg Psychiatry. 2000; 69:479-486. 
NOTES 